\title{
Centro de Atenção Psicossocial (CAPS): ações desenvolvidas em município de Minas Gerais, Brasil
}

\section{Psychosocial Care Center (CAPS): actions taken by a municipality of the state of Minas Gerais State, Brazil}

\author{
Tays Aparecida da Silva*1 \\ José Dionisio de Paula Júnior*2 \\ Ronaldo Chicre Araújo*3
}

Esta pesquisa teve como objetivo apresentar as ações desenvolvidas em Centro de Atenção Psicossocial [CAPS, modalidade II], no ano de 2015, em municipio de Minas Gerais. Trata-se de um estudo retrospectivo, descritivo exploratório, com base em fonte de dados secundários consultados no Tabulador para Windows [TABWIN]. Com isso, foi constatado que é possivel o CAPS desenvolver ações intersetoriais, multidisciplinares, de promoção e prevenção de agravos para a saúde mental, além de atenção à crise. Dessa forma, é importante estudos que apresentem discussões em torno do fortalecimento do protagonismo de usuários assistidos na esfera da saúde mental e a garantia de espaços participativos em toda a sociedade.

Palavras-chave: Centros de Atenção Psicossocial, procedimentos, psicologia, Saúde Mental

*1,2,3 Fundação Presidente Antônio Carlos- FUPAC (Ubá, MG, Brasil) 


\section{SAÚDE MENTAL}

\section{Introdução}

A lógica manicomial prediz a exclusão de um indivíduo do convívio social; diante disso, as internações em hospitais psiquiátricos promovem uma invisibilidade social com o enfraquecimento, fragilização e rompimento dos vínculos familiares. As ações dos hospitais psiquiátricos baseavam-se diretamente na cura do paciente, justificando a periculosidade do indivíduo com necessidade de institucionalização (Ferro, 2009; Torre \& Amarante, 2001; Lancetti \& Amarante, 2006).

Assim, nessa esfera de exclusão ocorre a mortificação da subjetividade, em que a doença mental é considerada um erro de razão e, portanto, o alienado não possuía direito a escolha (Amarante, 1995a; Lancetti \& Amarante, 2006). A atuação médica era centrada na doença, no quadro clínico e nos sintomas objetivos, desaparecendo todo o histórico e a individualidade do sujeito. Essa inserção para um mundo patológico, fora de um contexto social, permitia somente a medicina exercer o saber sobre o corpo do doente (Rotelli, 1990). Nesse contexto, surge um questionamento: os efeitos da cronicidade concebidos pela psiquiatria não são produção da própria institucionalização? Ora, em toda doença há a construção de uma subjetividade e por isso não se deve tratar somente o sintoma, mas sim o sujeito com uma percepção holística (Torre \& Amarante, 2001).

Dessa forma, fez-se necessária a criação de uma instituição que levasse em conta o sofrimento, existência em relação ao corpo social e rompesse com a conexão de causa e efeito empregado pela biomedicina clássica (Rotelli, 1990). Nesse sentido, Franco Basaglia inicia a ruptura com o saber psiquiátrico e desconstrução das relações entre instituições, práticas e saberes em detrimento dos direitos 
e cidadania dos pacientes, com a ideia de colocar a doença entre parêntese e ocupar-se do sujeito (Amarante, 1995b; Ferro, 2009). Nessa ruptura surge a necessidade de superar o modelo de humanização dos hospitais psiquiátricos, o dispositivo psiquiátrico de tutela, a diferenciação daquele que trata e do que é tratado, o lugar de desvalorização do louco de não poder falar de si mesmo, a exclusão e periculosidade presentes no imaginário social, chegando à máxima expressão de fechamento dos hospitais psiquiátricos e substituição por serviços territoriais (Amarante, 1995b; Lancetti \& Amarante, 2006).

Assim, percebemos a reforma psiquiátrica como um processo político e social complexo, composta de diversos atores e militâncias (Pitta, 2011). Esse cenário diz respeito ao processo de desinstitucionalização que não se reduz à desospitalização, pois não se restringe ao fechamento de hospitais psiquiátricos, mas a uma desconstrução de todo conceito de tratamento centrado na doença, violência e mortificação. Com essa visão, o doente torna sujeito desejante e político, construtor de projetos e subjetividade, além de protagonista de sua própria vida, e não apenas um objeto do saber psiquiátrico e corpo institucional, passando a ter direitos e assistência efetiva fora dos "cativeiros" (Amarante, 1995a; Torre \& Amarante, 2001).

Dessa forma, se não apresentar clareza de todas essas mudanças, além das instituições, existe o risco de continuidade para a lógica manicomial, mesmo com os novos conceitos territoriais (Torre \& Amarante, 2001), pois os serviços substitutivos não garantem a superação do "desejo de manicômio", descrito por Alverga e Dimenstein (2006) como um desejo que atinge usuários, familiares e a equipe multiprofissional, no sentido de classificar, dominar, oprimir, hierarquizar e controlar.

No Brasil, todo esse processo ganhou maior visibilidade no fim dos anos 1960, com o apoio de profissionais, usuários, familiares, sindicatos, associações e militantes dos Direitos Humanos (Pitta, 2011). Entretanto, em vários locais do Brasil foram surgindo novos métodos de intervenção, para a saúde mental, como a inauguração do primeiro CAPS, em 1987, na cidade de São Paulo, intitulado CAPS Professor Luiz da Rocha Cerqueira, e a intervenção na Casa de Saúde Anchieta, também em São Paulo, marcando de forma radical a desinstitucionalização no país (Pitta, 2011; Amarante, 1995b).

Atualmente os CAPS são o "carro-chefe" da Reforma Psiquiátrica, diferenciando-se do manicômio pela qualidade de suas respostas e dependência de toda a rede (Miranda, Oliveira \& Santos, 2014). Tem como objetivo ofertar cuidado para as pessoas com transtornos mentais, de forma territorializada, visando à organização da rede de serviços de saúde mental, a construção de 
projetos terapêuticos singulares, o suporte à saúde mental na Atenção Básica e unidades hospitalares, entre outras atividades. Sempre em busca de um modelo que priorize a reabilitação e a reintegração psicossocial do portador de transtorno mental, mediante acesso ao trabalho, lazer, exercício dos direitos civis e fortalecimento dos laços familiares (Ministério da Saúde, 2004; Luzio $\&$ Yasui, 2010).

Contudo, mediante tantas incumbências, os CAPS devem trabalhar de forma articulada com os demais serviços, pois não são os únicos responsáveis pelas intervenções em saúde mental. Cabe ressaltar suas inúmeras dificuldades devido às diversas tarefas e o déficit de apoio dos diversos setores que comportam o sistema de atenção a saúde no Brasil (Miranda, Oliveira $\&$ Santos, 2014). Diante disso, percebe-se que as transformações trouxeram intervenções mais complexas e interdisciplinares, para além do campo da saúde, exigindo uma reconstrução de práticas e saberes (Oliveira et al., 2014; Luzio \& Yasui, 2010).

Um avanço alcançado para a saúde mental foi a aprovação da Lei n. 10216 em 6 de abril de 2001, que dispõe sobre a proteção e direitos das pessoas portadoras de transtornos mentais e redireciona o seu modelo assistencial. Entre outras providências, essa Lei veta a internação de pacientes portadores de transtornos mentais em instituições de características asilares, onde visa a sua reinserção social e o tratamento em centros comunitários de saúde mental, preferencialmente (Brasil, 2001).

Posteriormente, no ano de 2011, foi instituída A Rede de Atenção Psicossocial [RAPS] pela Portaria n. 3088, tendo como objetivos ampliar $\mathrm{o}$ acesso à atenção psicossocial da população, promover a vinculação das pessoas com transtornos mentais e/ou com abuso de álcool e outras drogas e suas famílias aos pontos de atenção da rede e garantir a articulação e integração da rede, visando o acolhimento, acompanhamento contínuo e atenção às urgências. Sendo assim, os serviços de base territorial, a partir de um modelo de rede, que podem compor o cuidado de sujeitos com sofrimento psíquico são: Atenção Básica em Saúde (Unidade Básica de Saúde, Equipes de Atenção Básica para populações em situações específicas, Núcleo de Apoio à Saúde da Família e Centros de Convivência), Atenção Psicossocial Especializada (Centros de Atenção Psicossocial em suas modalidades), Atenção de Urgência e Emergência (Unidade de Pronto Atendimento [UPA], Serviço de Atendimento Móvel de Urgência [SAMU]), Atenção Residencial de Caráter Transitório (Unidade de Acolhimento Adulto, Unidade de Acolhimento Infanto-Juvenil e Comunidade Terapêutica), 
Atenção Hospitalar (leitos de saúde mental em hospital geral), Estratégias de Desinstitucionalização (Serviço Residencial Terapêutico e o Programa de Volta para Casa) e a Reabilitação Psicossocial (cooperativas sociais).

Diante de todos esses processos de normatização da assistência, a implantação da Rede de Atenção Psicossocial vem sendo realizada em todo território nacional, de modo não linear e simétrico (Pitta, 2011). Em levantamento realizado pelo Ministério da Saúde [MS], o Brasil tem denotado uma significativa expansão na implantação dos Centros de Atenção Psicossocial [CAPS], apresentando no ano de 1998 um total de 148 CAPS habilitados e, em dezembro de 2014, esse número aumenta para 2.209, revelando uma ampliação das ofertas de cuidado em serviços (Ministério da Saúde, 2015). Esses dados são considerados indicadores positivos, pois estudos apontam uma maior efetividade no tratamento de pessoas com transtornos mentais em serviços territoriais, se comparadas àquelas tratadas em privação de sua liberdade (Pitta, 2011). Além disso, os avanços na assistência à saúde mental ainda apresentam fragilidades e desafios a serem enfrentados (Tomasi et al., 2010; Oliveira et al., 2014; Luzio \& L'Abbate, 2009). Nesse contexto de fechamento dos hospitais psiquiátricos e sua substituição por serviços de base territorial, faz-se necessário analisar as ações dos CAPS frente ao complexo cenário brasileiro.

Com isso, este estudo tem como objetivo apresentar as ações desenvolvidas no Centro de Atenção Psicossocial [CAPS, modalidade II], no ano de 2015, em município de Minas Gerais, propulsor do cuidado em liberdade e articulador de uma rede integrada para a saúde mental.

\section{Métodos}

Trata-se de um estudo retrospectivo, descritivo exploratório, com base em fonte de dados secundários consultados no Tabulador para Windows [TABWIN], a fim de verificar os procedimentos realizados pelo CAPS II do município de Ubá, Minas Gerais, Brasil. Os dados foram coletados por discente do curso de graduação em Psicologia, da Fundação Presidente Antônio Carlos, entre os meses de abril e junho de 2016.

Dessa forma, na pesquisa foi utilizado o arquivo de definição "RAAS PSICOSSOCIAL.DEF", definindo como parâmetros de pesquisa: na linha, as ações realizadas; na coluna, o ano de atendimento; no incremento, as quantidades apresentadas e nas seleções disponíveis para filtro, o Cadastro Nacional 
de Estabelecimentos de Saúde [CNES] do CAPS executor. A pesquisa utilizou o arquivo do Registro de Ações Ambulatoriais de Saúde [RAAS], pois conforme a Nota Técnica n. 037/2016, ele é o instrumento de coleta para os procedimentos de CAPS e compreende o Boletim de Produção Ambulatorial Individualizado [BPA/I] e o Boletim de Produção Ambulatorial Consolidado [BPA/C]. Nesse contexto cabe enfatizar que, segundo a Portaria GM n. 3.462 de 11 de novembro de 2010, é de responsabilidade da gestão do estabelecimento a alimentação dos Bancos de Dados Nacionais do SCNES e do Sistema de Informação Ambulatorial [SIA/SUS] e a falta de registros e/ou atualização nesses sistemas pode determinar a suspensão de recursos federais de custeio dos estabelecimentos.

Além da Nota Técnica n. 037/2016 comprovar o funcionamento do CAPS por meio da alimentação regular dos procedimentos no SIA/SUS (RAAS, BPA-C e BPA-I), ela também solicita o esforço dos gestores e profissionais dos CAPS no sentido de se adequar às suas práticas visando a oferta de cuidado integral, promoção de protagonismo dos usuários, atuação intersetorial e comunitária, além de articulação com outros pontos da rede; acompanhar o relatório gerencial do SIA/SUS para verificar possíveis erros e problemas que podem comprometer a aprovação dos registros enviados; monitorar as ações oferecidas pelo serviço por meio do SIA/SUS (Tabnet ou Tabwin). Além disso, a mesma esclarece que a Portaria n. 854, de 22 de agosto de 2012, readequou os procedimentos e instrumentos de coleta de dados dos CAPS, valorizando aspectos como intersetorialidade, integralidade do cuidado e o papel da coordenação/matriciamento atribuído aos CAPS na RAPS, criando procedimentos como redução de danos, reabilitação psicossocial, promoção de protagonismo de usuários e outros.

A pesquisa também verificou a descrição de cada procedimento em separado, a fim de informar de forma precisa e clara qual a ação englo-bada em cada código. Para isso, foi realizada consulta ao Sistema de Gerenciamento da Tabela de Procedimentos, Medicamentos, Órteses, Próteses e Materiais Especiais do Sistema Único de Saúde [SIGTAB], pois a mesma serve de base para todos os sistemas ligados ao Sistema Único de Saúde [SUS], inclusive o Boletim de Produção Ambulatorial. Para tanto, foram utilizados os seguintes parâmetros: no grupo, o código 03 de procedimentos clínicos; no subgrupo, o código 01 de consultas/atendimentos/acompanhamentos e na forma de organização, o código 08 de atendimento/acompanhamento psicossocial. 
Assim, a fim de contextualizar o estudo e ampliar a visão do Estado na qual a cidade de Ubá está inserida, foram pesquisados os serviços habilitados que compõem a RAPS em Minas Gerais. E em relação à cidade de Ubá e ao CAPS II, foram verificados: os serviços cadastrados que compõem a RAPS do município e os profissionais atuantes no CAPS II, através do Sistema de Cadastro Nacional de Estabelecimentos de Saúde [SCNES]; a estimativa da população da cidade, com base nos dados do Instituto Brasileiro de Geografia e Estatística [IBGE]; e a atual inserção do município em projetos financiados pelo Ministério da Saúde.

A pesquisa atendeu todos os critérios éticos em acordo com a resolução 466/12 do Ministério da Saúde, por se tratar de informações públicas, não foi necessário a apreciação do estudo ao comitê de ética. Este estudo não apresenta conflito de interesses.

\section{Resultados}

Os dados demonstraram que os procedimentos realizados com maior frequência foram as "ações de atendimento individual", "acolhimento diurno" e "atendimento em grupo dos pacientes no CAPS". Entretanto, alguns pontos como "fortalecimento do protagonismo dos usuários", "acolhimento inicial" e "matriciamento das equipes dos pontos da urgência, emergência e serviços hospitalares", obtiveram uma menor frequência; conforme consta na tabela 1 .

Tabela 1 - Procedimentos do Centro de Atenção Psicossocial

\begin{tabular}{|cc|}
\hline \multicolumn{2}{|c|}{$\begin{array}{c}\text { Produção Ambulatorial de Procedimentos da Tabela Unificada Frequência por ano de } \\
\text { atendimento segundo Procedimento }\end{array}$} \\
\hline \multicolumn{1}{|c|}{ PROCEDIMENTOS } & Frequência/Ano \\
& 2015 \\
Atendimento em Oficina Terapêutica I - Saúde Mental & 201 \\
Atendimento em Oficina Terapêutica II - Saúde Mental & 729 \\
Atendimento Individual em psicoterapia & 152 \\
Acolhimento diurno de paciente em Centro de Atenção Psicossocial & 4182 \\
Atendimento individual de paciente em Centro de Atenção Psicossocial & 4332 \\
Atendimento em grupo de paciente em Centro de Atenção Psicossocial & 2690 \\
\hline
\end{tabular}




\section{SAÚDE MENTAL}

Atendimento familiar em Centro de Atenção Psicossocial

Acolhimento inicial por Centro de Atenção Psicossocial

Atendimento domiciliar para pacientes de Centro de Atenção Psicossocial

e/ou familiares

Ações de articulação de redes intra e intersetoriais

Fortalecimento do Protagonismo de Usuários de Centro de Atenção

Psicossocial e seus familiares

Práticas Corporais em Centro de Atenção Psicossocial

Práticas expressivas e comunicativas em Centro de Atenção Psicossocial

Atenção às situações de crise

Matriciamento de Equipes da Atenção Básica

Promoção de Contratualidade no território

Matriciamento de equipes dos pontos de atenção da urgência e emergência, e dos serviços hospitalares
110

2291

412

85

A fim de contextualizar esses dados, foram verificados os seguintes serviços habilitados em Minas Gerais: 178 CAPS, sendo que dos 853 municípios, somente 248 eram elegíveis para implantação, conforme os critérios populacionais, totalizando uma cobertura de 0,95 CAPS por 100 mil habitantes, o que é considerado um índice bom; 2 Equipes de Consultório na Rua, na modalidade I, 10 equipes na modalidade II e apenas 1 na modalidade III; 2 Unidades de Acolhimento Adulto e 2 Unidades de Acolhimento Infantil; 69 Serviços Residenciais Terapêuticos habilitados, perfazendo um total de 525 moradores, sendo que haviam 116 em funcionamento mas não habilitados; 42 hospitais de referência com leitos de saúde mental habilitados, totalizando 176 leitos; 623 beneficiários do Programa Volta para Casa e 77 iniciativas de trabalho de geração e renda do Componente de Reabilitação Psicossocial. Além disso, Minas Gerais ainda conta com 13 hospitais psiquiátricos e um total de 1798 leitos SUS nesses hospitais. Essa situação denota que a RAPS precisa se esforçar e organizar o cuidado em saúde mental visando às estratégias de desinstitucionalização (Ministério da Saúde, 2015), tabela 2. 
Tabela 2 - Descrição dos procedimentos realizados pelo Centro de Atenção Psicossocial

\section{PROCEDIMENTO DESCRIÇÃO}

\section{Oficina Terapêutica I}

Oficina Terapêutica II

Atendimento Individual em psicoterapia

\section{Acolhimento diurno de paciente}

\section{Atendimento individual}

\section{Atendimento em grupo}

\section{Atendimento familiar}

Acolhimento inicial

\section{Atendimento domiciliar}

Ações de articulação de redes intra e intersetoriais

Fortalecimento do Protagonismo
Atividade profissional em grupo (no mínimo 05 e no máximo 20 pacientes) de socialização, expressão e inserção social, com duração mínima de 2 horas executadas por profissional de nível médio.

Atividade profissional em grupo (no mínimo 5 e no máximo 20 pacientes) de socialização, expressão e inserção social.

Consiste no atendimento psicoterápico realizado por profissional de saúde mental.

Ação de hospitalidade diurna realizada nos CAPS como recurso do projeto terapêutico singular. Recorre ao afastamento do usuário das situações conflituosas, visando ao manejo de situações de crise motivado por sofrimentos decorrentes de transtornos mentais, incluídos aqueles por uso de drogas.

Atendimento direcionado à pessoa que comporte diferentes modalidades, responda às necessidades de cada um incluindo os cuidados de clínica geral.

Ações desenvolvidas coletivamente que explorem as potencialidades das situações grupais com variadas finalidades, como recurso para promover sociabilidade.

Ações voltadas para o acolhimento individual ou coletivo dos familiares e suas demandas, sejam elas decorrentes ou não da relação direta com os usuários.

Consiste no primeiro atendimento ofertado pelo CAPS para novos usuários, por demanda espontânea ou referenciada, incluindo as situações de crise no território.

Atenção prestada no local de morada da pessoa e/ou familiares.

Estratégias que promovam a articulação com outros pontos de atenção da rede de saúde, educação, justiça, assistência social, direitos humanos e outros, assim como os recursos comunitários presentes no território.

Atividades que fomentem a participação de usuários e familiares nos processos de gestão dos serviços e da rede. 


\begin{tabular}{|c|c|}
\hline Práticas corporais & $\begin{array}{l}\text { Estratégias ou atividades que favoreçam a percepção } \\
\text { corporal, a autoimagem, a coordenação psicomotora e os } \\
\text { aspectos somáticos e posturais da pessoa. }\end{array}$ \\
\hline $\begin{array}{l}\text { Práticas expressivas } \\
\text { e comunicativas }\end{array}$ & $\begin{array}{l}\text { Estratégias ou atividades que possibilitem ampliação do } \\
\text { repertório comunicativo e expressivo dos usuários. }\end{array}$ \\
\hline $\begin{array}{l}\text { Atenção às situações } \\
\text { de crise }\end{array}$ & $\begin{array}{l}\text { Ações desenvolvidas para o manejo das situações de crise, } \\
\text { entendidas como momentos do processo de acompanha- } \\
\text { mento dos usuários, nos quais conflitos relacionais geram } \\
\text { intenso sofrimento e desorganização. }\end{array}$ \\
\hline $\begin{array}{l}\text { Matriciamento da } \\
\text { Atenção Básica }\end{array}$ & $\begin{array}{l}\text { Apoio presencial sistemático às equipes de atenção básica } \\
\text { que oferte suporte técnico à condução do cuidado em saúde } \\
\text { mental através de discussões de casos e do processo } \\
\text { de trabalho. }\end{array}$ \\
\hline $\begin{array}{l}\text { Contratualidade no } \\
\text { território }\end{array}$ & $\begin{array}{l}\text { Acompanhamento de usuários em contextos reais de } \\
\text { vida - cenários de vida cotidiana. }\end{array}$ \\
\hline $\begin{array}{l}\text { Matriciamento da urgência } \\
\text { e emergência }\end{array}$ & $\begin{array}{l}\text { Apoio presencial sistemático às equipes dos pontos } \\
\text { de atenção da urgência e emergência. }\end{array}$ \\
\hline
\end{tabular}

Em relação ao município de Ubá, a cidade possui uma população estimada em 112.186 mil habitantes e possui os seguintes componentes da RAPS: 19 equipes de Estratégia de Saúde da Família [ESF] e 2 Núcleos de Apoio à Saúde da Família [NASF], na rede de atenção básica em saúde; 1 CAPS II e 1 CAPS AD III na atenção especializada em saúde mental; uma Base Descentralizada do SAMU na atenção de Urgência e Emergência; 6 leitos de saúde mental localizados na Casa de Saúde Padre Damião, na atenção hospitalar.

No quesito de formação e educação permanente, o município participou de dois projetos financiados pelo Ministério da Saúde, no âmbito da saúde mental. Um deles foi o projeto "Caminhos do Cuidado" que se configura na qualificação de profissionais, da rede de Atenção Básica à Saúde, a trabalharem em conjunto com saúde mental, levando em conta a realidade local de cada território. E também o projeto "Percurso Formativo na Rede de Atenção Psicossocial", sendo contemplado como rede visitante, no módulo de desinstitucionalização e sua rede receptora o município de Sorocaba/SP. O intercâmbio teve como objetivo a troca de experiências e saberes entre os profissionais, conforme o módulo de formação (Ministério da Saúde, 2015; Minas Gerais, 2015). 
Em relação ao CAPS II analisado, o serviço de gestão municipal possui uma equipe composta pelos seguintes profissionais: 1 administrador, 1 artista de artes visuais, 1 técnico de enfermagem, 1 assistente social, 2 assistentes administrativos, 2 médicos psiquiatras, 2 enfermeiros e 6 psicólogos clínicos, sendo realizado atendimento ambulatorial de demanda espontânea ou referenciada.

\section{Discussão}

Analisando as ações realizadas, no ano de 2015, pelo CAPS II do município de Ubá, a Portaria n. 336/2002 e a Resolução SES/MG n. 4946/2015, podemos perceber que há uma conformidade entre as ações estabelecidas pelas últimas e as oferecidas pelo CAPS analisado, sendo elas: visitas domiciliares, atendimento à família, atividades comunitárias com objetivo de integração na família e comunidade, oficinas terapêuticas, supervisão e capacitação das equipes de atenção básica, supervisão de unidades hospitalares, desenvolvimento de ações intersetoriais, elaboração de projeto terapêutico singular e atendimento de usuários em crise.

Assim, podemos sinalizar que os procedimentos realizados com maior frequência no CAPS são fundamentais, pois os usuários inseridos nessas ações, em um regime mais intensivo, provavelmente tenderão a diminuir a medicação, reduzir as crises e, consequentemente, as intercorrências que levam a internações hospitalares (Tomasi et al., 2010).

Portanto, esses avanços denotam que é possível ter uma nova forma de assistência, com cuidado individualizado, práticas intersetoriais, promoção do cuidado no território e inserção social que evitam a reprodução das marcas da cultura asilar e das práticas criadoras de cronicidades (Neto \& Amarante, 2013).

Entretanto, no que diz respeito à realização de apenas uma ação voltada para o fortalecimento do protagonismo dos usuários no CAPS analisado, podemos considerar que a participação social é muito recente no nosso país, garantida legalmente no ano de 1990 pela Lei n. 8.142. Além disso, encontra-se a dificuldade de fomentar o protagonismo de um "usuário de saúde mental" marcado pela exclusão e incapacidade de gerir sua própria vida. O que indica a necessidade de mais debates e pesquisas em torno da participação que é possível e desejável nessa área, no âmbito do SUS. Em pesquisa realizada no Rio Grande do Sul, foi apontado que há processos que constrangem o protagonismo e mantêm a passividade dos usuários, com certo enrijecimento e hierarquização entre os profissionais e os usuários; entretanto, também foi relatado 


\section{SAÚDE MENTAL}

que existem espaços participativos potentes, mas que ainda não foram percebidas as riquezas que produzem. De qualquer forma, o indicativo analisado nos faz apurar a necessidade de espaços participativos dos usuários, tanto para que eles sejam protagonistas de suas próprias vidas quanto para que a sociedade seja capaz de acolher a diferença radical da loucura (Costa \& Paulon, 2012).

Em relação ao acolhimento inicial, um estudo verificou que há dificuldades dos profissionais em acolherem e escutarem o sofrimento alheio, levando muitas vezes a uma prescrição de medicamentos como forma de diminuir a angústia ou o choro do usuário (Minóia \& Minozzo, 2015). Porém, outro estudo demonstrou que os profissionais não possuem a mesma percepção sobre a definição de acolhimento; alguns restringiram o conceito a uma mera triagem (Scheibel \& Ferreira, 2011). Dessa forma, interroga-se até que ponto o acolhimento inicial no CAPS analisado foi realizado de forma insuficiente ou se houve um sub-registro dessa ação por razões de equívocos na caracterização do procedimento.

No que diz respeito às ações de matriciamento, podemos considerar que sua realização de forma efetiva promove uma organização da atenção à saúde no SUS, atuação multiprofissional, ligação entre os diversos serviços e consequente ampliação e potencialização do cuidado perante os casos clínicos em saúde mental (Silva \& Dimenstein, 2014; Pinto et al., 2012). Em relação a essa ação na urgência e emergência, podemos ponderar que o SAMU tem apresentado dificuldades em cumprir o seu papel mediante as solicitações de apoio à equipe do CAPS (Silva \& Dimenstein, 2014).

Diante desses entraves os usuários podem se deparar com fragmentações no fluxo da atenção que, muitas vezes, podem ser somados à baixa cobertura dos serviços de saúde que caracterizam ainda mais a fragmentação do cuidado (Pinto et al., 2012).

Cabe ressaltar que existe uma dificuldade em avaliar a totalidade da qualidade oferecida pelos serviços de saúde mental, pois há aspectos subjetivos envolvidos em todo o processo, que perpassam as análises dos procedimentos realizados pelos serviços (Oliveira et al., 2014; Luzio \& Yasui, 2010).

\section{Considerações finais}

Foi possível compreender a proposta da Política Nacional de Saúde Mental, pois as informações, descritas no estudo, contextualizaram as diversas ações do CAPS, no desenvolvimento de ações intersetoriais, 
multidisciplinares, de promoção/prevenção de agravos e assistência aos usuários em crise, no qual o CAPS possui importantes atribuições na superação da lógica manicomial que está inserida no imaginário social.

Dessa forma, as abordagens sobre a desinstitucionalização e a saúde mental precisam ser mais amplamente discutidas com debates, políticas públicas e promoção de pesquisas.

\section{Referências}

Alverga, A. R., \& Dimenstein, M. (2006). A reforma psiquiátrica e os desafios da desinstitucionalização da loucura. Interface - Comunicação, Saúde, Educação, 10(20), 299-316.

Amarante, P. (1995a). Novos sujeitos, novos direitos: o debate em torno da reforma psiquiátrica. Caderno de Saúde Pública, 11(3), 491-494.

Amarante, P. (1995b). Loucos pela vida: a trajetória da reforma psiquiátrica no Brasil (2 $2^{\mathrm{a}}$ ed.). Rio de Janeiro, RJ: Fiocruz.

Brasil (2001). Lei Federal n. 10.216, de 6 de abril de 2001. Dispõe sobre a proteção e direitos das pessoas portadoras de transtornos mentais e redireciona o modelo assistencial em saúde mental. Brasília, DF. Recuperado em 7 de maio de 2016, de: $<$ http://www.planalto.gov.br/ccivil_03/leis/LEIS_2001/L10216.htm>.

Costa, D. F. C., \& Paulon, S. M. (2012). Participação social e protagonismo em saúde mental: a insurgência de um coletivo. Saúde em Debate, 36(95), 572-582.

Ferro, L. F. (2009). Trabalho territorial em hospitais psiquiátricos - construindo no presente um futuro sem manicômios. Psicologia e Ciência da Profissão, 29(4), 752-767.

Lancetti, A., \& Amarante, P. (2006). Saúde Mental e Saúde Coletiva. In G. W. S. Campos, M. C. S. Minayo, M. Akerman, M. Drumond Jr., \& Y. M. Carvalho (Orgs.). Tratado de Saúde Coletiva (pp. 615-633). São Paulo, SP/Rio de Janeiro, RJ: Hucitec, Fiocruz.

Luzio, C. A., \& L'Abatte, S. (2009). A atenção em saúde mental em municípios de pequeno e médio portes: ressonâncias da reforma psiquiátrica. Ciência \& Saúde Coletiva, 14(1), 105-116.

Luzio, C. A., \& Yasui, S. (2010). Além das portarias: desafios da política de saúde mental. Psicologia em Estudo, 15(1), 17-26.

Minas Gerais. (2015). Projeto Caminhos do Cuidado em Minas Gerais: Relatório Final. Recuperado em 4 jul., 2016, de: <http://www.caminhosdocuidado.org>.

Ministério da Saúde. (2004). Saúde mental no SUS: os Centros de Atenção Psicossocial. Recuperado em 16 maio 2016, de: <www.saude.gov.br/bvs/ saudemental>. 


\section{SAÚDE MENTAL}

Ministério da Saúde. (2015). Saúde Mental em Dados. Recuperado em 9 maio 2016, de: <www.saude.gov.br/bvs/saudemental $>$.

Minóia, N. P., \& Minozzo, F. (2015). Acolhimento em saúde mental: operando mudanças na atenção primária à saúde. Psicologia, Ciência e Profissão, 35(4), 1340-1349.

Miranda, L., Oliveira, T. F. K., Santos, C. B. T. (2014). Estudo de uma Rede de Atenção Psicossocial: Paradoxos e Efeitos da Precariedade. Psicologia, Ciência e Profissão, 34(3), 592-611.

Neto, M. L. A., \& Amarante, P. D. C. (2013). O acompanhamento terapêutico como estratégia de cuidado na atenção psicossocial. Psicologia, Ciência e Profissão, 33(4), 964-975.

Nota técnica n. 037 (2016, 08 abril). Solicita mobilização para melhoria dos registros de procedimentos dos Centros de Atenção Psicossocial no SIA-SUS. Diário Oficial da União, Brasília-DF.

Oliveira, M. A. F., Cestari, T. Y., Pereira, M. O., Pinho, P. H., Gonçalves, R. M. D. A., \& Claro, H. G. (2014). Processos de avaliação de serviços de saúde mental: uma revisão integrativa. Saúde Debate, 38(101), 368-378.

Pinto, A. G. A., Jorge, M. S. B., Vasconcelos, M. G. F., Sampaio, J. J. C., Lima, G. P., Bastos, V. C., \& Sampaio, H. A. C. (2012). Apoio matricial como dispositivo do cuidado em saúde mental na atenção primária: olhares múltiplos e dispositivos para resolubilidade. Ciência \& Saúde Coletiva, 17(3), 653-660.

Pitta, A. M. F. (2011). Um balanço da Reforma Psiquiátrica Brasileira: instituições, atores e políticas. Ciência \& Saúde Coletiva, 16(12), 4579-4589.

Portaria n. 336 (2002, 19 fevereiro). Regulamenta o funcionamento dos Centros de Atenção Psicossocial. Diário Oficial da União, Brasília-DF.

Portaria n. 3462 (2010, 11 novembro). Estabelece critérios para alimentação dos Bancos de Dados Nacionais dos Sistemas de Informação da Atenção à Saúde. Diário Oficial da União, Brasília-DF.

Portaria n. 3088 (2011, 23 dezembro). Institui a Rede de Atenção Psicossocial para pessoas com sofrimento ou transtorno mental e com necessidades decorrentes do uso de crack, álcool e outras drogas, no âmbito do Sistema único de Saúde. Diário Oficial da União, n. 251, Brasília-DF.

Portaria n. 854 (2012, 22 agosto). Altera a Tabela de Procedimentos, Medicamentos, Órteses, Próteses e Materiais Especiais do Sistema Único de Saúde. Diário Oficial da União, Brasília-DF.

Resolução n. 4946 (2015, 13 outubro). Estabelece as normas gerais de adesão, execução, acompanhamento, controle e avaliação do processo de concessão do incentivo financeiro mensal para os Centros de Atenção Psicossocial em suas 
diversas modalidades. Secretaria de Estado da Saúde de Minas Gerais, Belo Horizonte-MG.

Rotelli, F. (1990). A instituição inventada. In F. Rotelli., O. Leonardis, D. Mauri, \& C. Risio. Desinstitucionalização. São Paulo, SP: Hucitec.

Scheibel, A., \& Ferreira, L. H. (2011). Acolhimento no CAPS: Reflexões acerca da assistência em saúde mental. Revista Baiana de Saúde Pública, 35(4), 966-983.

SCNES - Sistema Cadastro Nacional de Estabelecimento de Saúde (n.d.). Estabelecimento de Saúde do Município de Ubá. Recuperado em 17 maio 2016, de: <http://cnes2.datasus.gov.br/Lista_Es_Municipio. asp? VEstado=31\&VCodMunicipio=316990\&NomeEstado=MINAS $\% 20$ GERAIS>.

SIGTAB - Sistema de Gerenciamento da Tabela de Procedimentos, Medicamentos, Órteses, Próteses e Materiais Especiais do Sistema Único de Saúde (n.d.). Consulta de Procedimentos. Recuperado em 18 maio 2016, de: <http://sigtap. datasus.gov.br/tabela-unificada/app/sec/inicio.jsp $>$.

Silva, M. L. B, \& Dimenstein, M. D. B. (2014). Manejo da crise: encaminhamento e internação psiquiátrica em questão. Arquivos Brasileiros de Psicologia, 66(3), $31-46$.

TabWin - Tabulador para Windows (n.d.). Produção Ambulatorial de Procedimentos da Tabela Unificada. Recuperado em 19 maio 2016, de arquivo C: $\backslash$ TabWin $\backslash$ SIA $\backslash$ PA*.DBC

Tomasi, E., Facchini, L. A., Piccini, R. X., Thumé, E., Silva, R. A., Gonçalves, H., \& Silva, S. M. (2010). Efetividade dos Centros de Atenção Psicossocial no cuidado a portadores de sofrimento psíquico em cidade de porte médio do Sul do Brasil: uma análise estratificada. Caderno de Saúde Pública, 26(4), 807-815.

Torre, E. H. G., \& Amarante, P. (2001). Protagonismo e subjetividade: a construção coletiva no campo da saúde mental. Ciência \& Saúde Coletiva, 6(1), 73-85.

\section{Resumos}

(Psychosocial Care Center [CAPS]: actions taken by a municipality of the state of Minas Gerais State, Brazil)

This paper describes actions taken by a Psychosocial Care Center (CAPS, type II) in 2015, in a municipality of the state of Minas Gerais, Brazil. We conducted a retrospective, descriptive-exploratory study based on secondary data source accessed by means of Tab for Windows (TabWin), which led to the conclusion that the CAPS is fully able to develop intersectoral, multidisciplinary actions that aim to promote mental health, 


\section{SAÚDE MENTAL}

prevent aggravation and support people to tackle crisis situations. We also highlight how essential studies are that provide discussions on how to increase the participation of users in the mental health sector and to promote participatory spaces in society as a whole.

Key words: Psychosocial Care Centers, procedures, psychology, Mental Health

(Centre de soins psychosociaux [CAPS]: actions développées dans une municipalité de l'état de Minas Gerais, Brésil)

Cet article présente des actions développées en 2015 dans un centre de soins psychosociaux (CAPS, modalité II) d'une municipalité de l'état de Minas Gerais, Brésil. Il s'agit d'une étude rétrospective, descriptive et exploratoire basée sur des sources de données secondaires qui ont été consultée à l'aide du logiciel Tab for Windows (TabWin). Nous arrivons à la conclusion que le CAPS est apte à développer des actions intersectorielles et multidisciplinaires visant à la promotion de la santé mentale, à la prévention de facteurs d'aggravation, ainsi qu'à la gestion de crises. Les études qui discutent l'amélioration du rôle des patients dans le domaine de la santé mentale sont donc fondamentales, ainsi que la garantie d'espaces participatifs de la société en général.

Mots clés: Centre de soins psychosociaux (CAPS), procédures, psychologie, Santé Mentale

(Centro de Atención Psicosocial [CAPS]: acciones desarrolladas en el municipio de Minas Gerais, Brasil)

Esta investigación tuvo como objetivo presentar las acciones desarrolladas en el Centro de Atención Psicosocial (CAPS, modalidad II), en el año 2015, en el municipio de Minas Gerais. Se trata de un estudio retrospectivo, descriptivo y exploratorio basado en fuentes de datos secundarios, consultadas en TabWin. Con esto, se constató que es posible que el CAPS desarrolle acciones intersectoriales, multidisciplinares, de promoción y prevención de riesgos para la salud mental, además de la atención de las crisis. De esta manera, son importantes los estudios que presenten discusiones sobre el fortalecimiento del protagonismo de los pacientes asistidos en el ámbito de la salud mental y la garantía de espacios participativos en toda la sociedad.

Palabras clave: Centros de Atención Psicosocial [CAPS], procedimientos, psicología, Salud Mental

(Psychosoziale Betreuungszentren (CAPS): Durchführung von Aktionen in einer Gemeinde von Minas Gerais, Brasilien)

Dieser Artikel stellt Aktionen vor, die 2015 in einem psychosozialen Betreuungszentrum (CAPS, Typ II) in einer Gemeinde des Staates Minas Gerais (Brasilien) entwickelt wurden. Durchgeführt wurde eine deskriptive, explorative und retrospektive Studie anhand von sekundären Datenquellen, die mittles Tab for Windows (TabWin) konsultiert wurden. Es wurde festgestellt, dass das CAPS in der Lage ist, sektorübergreifende und 
multidisziplinäre Aktionen durchzuführen, die dazu dienen, die psychische Gesundheit zu fördern, die Verschlimmerung von Erkrankungen zu verhindern, sowie den Umgang mit Krisen zu vermitteln. Abschließend unterstreicht dieser Artikel die grundlegende Rolle von Studien, welche die Teilnahme der betreuten Benutzer im Bereich der psychischen Gesundheit diskutieren und unterstützen und wie wichtig es ist, die Teilnahme der Gesellschaft an diesen Problemen zu sichern.

Schlüsselwörter: Psychosoziale Betreuungszentren, Behandlung, Psychologie, geistige Gesundheit

Citação/Citation: Silva, T. A. da, Paula Junior, J. D., Araújo, R. C. (2018, junho). Centro de Atenção Psicossocial [CAPS]: ações desenvolvidas em município de Minas Gerais, Brasil. Revista Latinoamericana de Psicopatologia Fundamental, 21(2), 346-363. http://dx.doi. org/10.1590/1415-4714.2018v21n2p346.8

Editores do artigo/Editors: Profa. Dra. Ana Cristina Costa Figueiredo e Profa. Dra. Andrea Máris Campos Guerra.

Recebido/Received: 13.11.2017/ 11.13.2017 Aceito/Accepted: 10.2.2018 / 2.10.2018

Copyright: (C) 2009 Associação Universitária de Pesquisa em Psicopatologia Fundamental/ University Association for Research in Fundamental Psychopathology. Este é um artigo de livre acesso, que permite uso irrestrito, distribuição e reprodução em qualquer meio, desde que o autor e a fonte sejam citados / This is an open-access article, which permits unrestricted use, distribution, and reproduction in any medium, provided the original authors and sources are credited.

Financiamento/Funding: Os autores declaram não terem sido financiados ou apoiados / The authors have no support or funding to report.

Conflito de interesses/Conflict of interest: Os autores declaram que não há conflito de interesses / The authors have no conflict of interest to declare. 


\section{SAÚDE MENTAL}

\section{Tays Aparecida da Silva}

Psicóloga. Fundação Presidente Antônio Carlos - FUPAC.

Rua João Rodrigues de Andrade, 165 - Boa Vista

36500-000 Ubá, MG, Brasil

tays.moura@yahoo.com.br.

\section{José Dionísio de Paula Júnior}

Enfermeiro. Doutor em Ciências Biomédicas. Docente do curso de graduação em Psicologia da Fundação Presidente Antônio Carlos - FUPAC.

Rua João Rodrigues de Andrade, 165 - Boa Vista

36500-000 Ubá, MG, Brasil

dionisiodepaula@yahoo.com.br.

\section{Ronaldo Chicre Araújo}

Psicólogo. Doutor em Ciência da Religião. Docente do curso de graduação em Psicologia da Fundação Presidente Antônio Carlos - FUPAC.

Rua João Rodrigues de Andrade, 165 - Boa Vista

36500-000 Ubá, MG, Brasil

rchicre@hotmail.com.

This is an open-access article, which permits unrestricted use, distribution,

(cC) BY-NC and reproduction in any medium for non-commercial purposes provided the original authors and sources are credited. 